\title{
Coherent Atom Interactions Mediated by Dark-State Polaritons
}

\author{
A. André ${ }^{1}$, L.-M. Duan ${ }^{2}$ and M.D. Lukin ${ }^{1}$ \\ ${ }^{1}$ ITAMP, Harvard-Smithsonian Center for Astrophysics and \\ Physics Department, Harvard University, Cambridge, MA 02138 \\ ${ }^{2}$ Institute for Theoretical Physics, University of Innsbruck, Austria.
}

(October 28, 2018)

\begin{abstract}
We suggest a technique to induce effective, controllable interactions between atoms that is based on Raman scattering into an optical mode propagating with a slow group velocity. The resulting excitation corresponds to the creation of spin-flipped atomic pairs in a way that is analogous to correlated photon emission in optical parametric amplification. The technique can be used for fast generation of entangled atomic ensembles, spin squeezing and applications in quantum information processing.

PACS numbers 03.67.-a, 42.50.-p, 42.50.Gy
\end{abstract}

The intriguing possibility for controlled manipulation of interacting quantum systems is the basis for a number of exciting developments in the field of quantum information science [1]. These are expected to have an impact in a broad area ranging from quantum computation and quantum communication [2 to precision measurements [3] and controlled modeling of complex quantum phenomena 《4].

This Letter describes a new technique to induce effective coherent interactions between atoms in metastable states. The technique is based on a resonantly enhanced nonlinear process involving Raman scattering into a "slow" optical mode [5], which creates a pair of spin-flipped atom and slowly propagating coupled excitation of light and matter (dark-state polariton). When the group velocity of the polariton is reduced to zero [6.7, this results in pairs of spin flipped atoms.

The present phenomenon of spin pair creation exhibits strong similarities with optical parametric amplification (OPA), in which pairs of photons are generated that possess non-classical correlations in photon number, quadrature component fluctuations or in polarization states [8]. In direct analogy, the present technique is capable of generating non-classically correlated atomic ensembles and entangled spin excitations. The latter can easily be converted into corresponding states of photon wavepackets "on demand", which makes the present approach most suitable for implementing protocols in quantum information processing that require a combination of deterministic sources of entangled states and long-lived quantum memory [9,10].

The present technique can also be viewed as a new mechanism for coherent "collisions" [11] between atoms mediated by light. In particular, the case when atomic pairs are excited into two different levels (as e.g. in Fig.1a) closely resembles coherent spin-changing interactions that occur in degenerate atomic samples [12], whereas the case when atomic pairs are stimulated into identical states (Fig.1b) is reminiscent of dissociation of a molecular condensate [13]. To put this analogy in perspective we note that the rate of the present optically induced process can exceed that of weak interatomic interactions by orders of magnitude. Therefore the present work may open up interesting new possibilities for studying many-body phenomena of strongly interacting atoms.

Before proceeding we note that a number of proposals have been made for generating entangled states of atomic ensembles and resulting in so-called spin squeezed states. Some are based on interatomic interactions at ultra-cold temperatures [14], whereas others involve mapping the states of non-classical light fields into atoms [15], QND measurements of spins 16] with light and Rydberg blockade 17. In contrast to these mechanisms the present approach does not require coherence of the atomic motion or sources of non-classical light and is completely deterministic thereby significantly simplifying possible experimental realizations. We further show that the present technique can be made robust with respect to realistic decoherence processes such as spontaneous emission and leakage of slow photons from the medium. We also note the work of Franson and co-workers 18] on quantum logic based on so-called "photon-exchange" interaction. However further analysis 19] showed that these mechanisms do not result in a non-linear interaction.

We consider a system of $N$ atoms (Fig.1) interacting with two classical driving fields and one quantized mode that is initially in a vacuum state. Relevant atomic sublevels include two manifolds of metastable states (e.g hyperfine sublevels of electronic ground state) and excited states that might be accessed by optical transitions. The atoms are initially prepared in their ground states $|g\rangle$. One of the classical fields (Rabi frequency $\Omega_{1}$ ) is detuned from the atomic resonance by an amount roughly equal to the frequency splitting between ground state manifolds. The other (Rabi frequency $\Omega_{2}$ ) is resonant with an atomic transition $\left|b_{2}\right\rangle \rightarrow\left|a_{2}\right\rangle$. The quantized field can be involved in two Raman transitions corresponding to Stokes and anti-Stokes processes. Whereas the former corresponds to the usual Stokes scattering in the forward direction, the latter establishes an Electromagnetically Induced Transparency (EIT) and slows down its group 
velocity. The pair excitation can be viewed as resulting from quantized photon exchange between atoms (Fig.2) in a two-step process. The first flipped spin is created due to Stokes Raman scattering, which also results in photon emission in a corresponding Stokes mode. In the presence of EIT, this photon is directly converted into a dark-state polariton which becomes purely atomic when the group velocity is reduced to zero. This implies that atomic spins are always flipped in pairs. In Fig.1a the two final states involved in Raman transitions are different and atomic pairs in different states are created. In Fig.1b the final states of the two Raman processes are identical, in which case atomic pairs in the same state result.

In what follows we will focus on a system (Fig.1a) involving two atomic modes. Consideration of the scheme of Fig.1b proceeds along the same lines. For conceptual simplicity we here assume that the quantized field corresponds to a single mode of a running-wave cavity with a creation operator $\hat{a}^{\dagger}$ and atom-field coupling constants $g_{1}$ and $g_{2}$. Generalization to multi-mode i.e. travelling wave configuration is straightforward. The interaction Hamiltonian for the system of $\mathrm{N}$ atoms and light can be split into two parts $H=H_{\text {ram }}+H_{\text {res }}$, which are given by:

$$
\begin{aligned}
H_{\text {ram }}= & -\hbar \Delta \Sigma_{a 1 a 1}-\hbar \delta_{1} \Sigma_{b_{1} b_{1}} \\
& +\left[\hbar \Omega_{1} \Sigma_{g a 1}+\hbar g_{1} a^{\dagger} \Sigma_{b_{1} a 1}+\text { h.c. }\right], \\
H_{\text {res }}= & \hbar \delta_{2} \Sigma_{b_{2} b_{2}}+\hbar \delta_{2} \Sigma_{a_{2} a_{2}} \\
& +\left[\hbar g_{2} a^{\dagger} \Sigma_{g a 2}+\hbar \Omega_{2} \Sigma_{b_{2} a 2}+\text { h.c. }\right],
\end{aligned}
$$

where $\Sigma_{\mu \nu}=\sum_{i}|\mu\rangle_{i i}\langle\nu|$ are collective atomic operators corresponding to transitions between atomic states $|\mu\rangle,|\nu\rangle, \Delta$ is the detuning of the classical field $\Omega_{1}$ from the single-photon transition $|g\rangle \rightarrow\left|a_{1}\right\rangle, \delta_{1}$ and $\delta_{2}$ are the two-photon detunings from the $|g\rangle \rightarrow\left|b_{1}\right\rangle$ and $|g\rangle \rightarrow\left|b_{2}\right\rangle$ transitions respectively as shown in Fig.1.

In the limit of large detuning $\Delta$ and ignoring twophoton detunings for the moment, the Hamiltonian $H_{\text {ram }}$ describes an off-resonant Raman scattering. After a canonical transformation corresponding to adiabatic elimination of the excited state $H_{\text {ram }}$ becomes equivalent to:

$$
H_{\text {ram }}=\hbar \sqrt{N} \frac{g_{1}^{*} \Omega_{1}}{\Delta} a S_{1}+\text { h.c. },
$$

where we disregarded the light shift $\delta_{L}=\Omega_{1}^{2} / \Delta$ and introduced $S_{1}=1 / \sqrt{N} \Sigma_{g b_{1}}$. The light shift $\delta_{L}$ can be easily compensated by re-defining the energy of the atomic levels and will be disregarded in the remainder of this Letter. The resonant part of the Hamiltonian $H_{\text {res }}$ is best analyzed in terms of dark and bright-state polaritons

$$
P_{D}=\frac{\Omega_{2} a-g_{2} \sqrt{N} S_{2}}{\sqrt{g_{2}^{2} N+\Omega_{2}^{2}}}
$$

$$
P_{B}=\frac{g_{2} \sqrt{N} a+\Omega_{2} S_{2}}{\sqrt{g_{2}^{2} N+\Omega_{2}^{2}}},
$$

which are superpositions of photonic and atomic excitations, with $S_{2}=1 / \sqrt{N} \Sigma_{g b_{2}}$. In particular, $H_{\text {res }}$ has an important family of dark-states:

$$
\left|D^{n}\right\rangle \sim\left(P_{D}^{\dagger}\right)^{n}|g\rangle|\mathrm{vac}\rangle
$$

with zero eigenenergies. Note that all other eigenstates of $H_{\text {res }}$ have, in general, non-vanishing interaction energy. Under conditions of Raman resonance and sufficiently slow excitation ("adiabatic condition") the Stokes photons emitted by Raman scattering, Eq.(3), will therefore couple solely to the dark-states (5). In this case the evolution of the entire system is described by an effective Hamiltonian:

$$
H_{e f f}=\hbar \xi\left(P_{D} S_{1}+S_{1}^{\dagger} P_{D}^{\dagger}\right),
$$

with $\xi=\Omega_{1} \Omega_{2} / \Delta \times g_{1}^{*} \sqrt{N} / \sqrt{\left|g_{2}\right|^{2} N+\Omega_{2}^{2}}$. The Hamiltonian (6) describes the coherent process of generation of pairs involving polaritons and spin-flipped atoms. Note that for a small number of excitations the spin waves and polaritons obey bosonic commutation relations and this Hamiltonian is formally equivalent to that describing optical parametric amplification (OPA) of two modes [8]. This is also analogous to the "counter-twisting" model of Ref. [21], which is known to result in maximal spin squeezing for large number of excitations.

We now consider the scenario in which the system is evolving for a time $\tau$ under the Hamiltonian $H_{\text {eff }}$, after which both fields are turned off. If the procedure is adiabatic upon turn-off of the coupling fields $\Omega_{1,2}$ the polaritons are converted into pure spin excitations $P_{D} \rightarrow S_{2}$. Hence the entire procedure will correspond to the following state of the system:

$$
\begin{aligned}
|\Psi\rangle= & \frac{1}{\cosh \xi \tau} \sum_{n}(\tanh \xi \tau)^{n} \frac{1}{n !}\left(P_{D}^{\dagger}\right)^{n}\left(S_{1}^{\dagger}\right)^{n}|g\rangle|\mathrm{vac}\rangle \\
& \rightarrow \frac{1}{\cosh \xi \tau} \sum_{n}(\tanh \xi \tau)^{n}\left|n_{b_{1}}, n_{b_{2}}\right\rangle|\mathrm{vac}\rangle .
\end{aligned}
$$

Here $\left|n_{b_{1}}, n_{b_{2}}\right\rangle=1 / n !\left(S_{2}^{\dagger}\right)^{n}\left(S_{1}^{\dagger}\right)^{n}|g\rangle$ are Dicke-like symmetric states of atomic ensemble and we assumed $n_{b_{1}, b_{2}} \ll N$. For non-zero $\xi \tau$ this state describes an entangled state, for which relative fluctuations between the two modes decreases exponentially to values well below the standard quantum limit (SQL) corresponding to uncorrelated atoms.

The above analysis only includes the interaction with a single (forward-propagating) quantized radiation mode and neglects decoherence processes. We now take into account realistic decoherence mechanisms such as spontaneous emission from the excited states in all directions and decay of the cavity mode with a rate $\kappa$. The evolution of atomic operators is then described by HeisenbergLangevin equations: 


$$
\dot{\Sigma}_{\mu \nu}=-\gamma_{\mu \nu} \Sigma_{\mu \nu}+\frac{i}{\hbar}\left[H, \Sigma_{\mu \nu}\right]+F_{\mu \nu},
$$

where $\gamma_{\mu \nu}$ is a decay rate of coherence $\mu \rightarrow \nu$ and $F_{\mu \nu}$ are associated noise forces. The latter have zero average and are $\delta$-correlated with associated diffusion coefficients that can be found using the Einstein relations. The cavity mode obeys the equation of motion:

$$
\dot{a}=-\kappa a-i g_{1} \Sigma_{b_{1} a_{1}}-i g_{2} \Sigma_{g a_{2}}+F_{a}(t) .
$$

We proceed by adiabatic elimination of optical polarizations associated with Stokes emission. To this end we assume large single-photon detuning $\Delta \gg \gamma$ and to first order in $\hat{a}$ we obtain the following equations of motion for the metastable coherences

$$
\begin{aligned}
& \dot{S}_{1}^{\dagger}=-\left[\bar{\gamma}_{g b}+i \delta_{1}\right] S_{1}^{\dagger}+i \frac{g_{1}^{*} \sqrt{N} \Omega_{1}}{\Delta} a+\bar{F}_{S_{1}}^{\dagger} \\
& \dot{S}_{2}=-\left[\bar{\gamma}_{g b}+i \delta_{2}\right] S_{2}-i\left(\Omega_{2} / \sqrt{N}\right) \Sigma_{g a_{2}}+\bar{F}_{S_{2}}
\end{aligned}
$$

where $\bar{\gamma}_{g b}=\gamma_{g b}+\gamma_{L}$ and $\gamma_{L}=\gamma_{a g}\left|\Omega_{1}\right|^{2} / \Delta^{2}$ is the optical pumping rate. The ground state coherence thus decays at a rate modified by isotropic spontaneous emission from the excited state (we consider the case $\gamma_{L} \gg \gamma_{g b}$ ).

To treat the resonant EIT-like interaction we first rewrite the equations of motion in terms of the dark and bright polariton operators (4) and proceed to adiabatically eliminate the optical coherence $\Sigma_{g a_{2}}$ and the bright state polariton $P_{B}$. In the relevant limit when $\left|g_{2}\right|^{2} N / \gamma_{a g} \kappa \gg 1$ and when $\eta=\left|g_{2}\right|^{2} N /\left|\Omega_{2}\right|^{2}$ the ratio of vacuum light velocity to group velocity is large $\eta \gg 1$, we find:

$$
\begin{aligned}
& \dot{P}_{D}=-\left[\frac{\kappa+\eta\left(\gamma_{L}+i \delta_{2}\right)}{1+\eta}\right] P_{D}+i \xi S_{1}^{\dagger}+\tilde{F}_{D}(t), \\
& \dot{S}_{1}^{\dagger}=\left[\frac{\left|g_{2}\right|^{2}}{\left|g_{1}\right|^{2}} \gamma_{L}-\gamma_{L}-i \delta_{1}\right] S_{1}^{\dagger}-i \xi P_{D}+\tilde{F}_{S_{1}}^{\dagger}(t) .
\end{aligned}
$$

We note that cavity losses are strongly suppressed in the limit $\eta \gg 1$ : subsequent to the large group velocity reduction [5], the polariton is almost purely atomic and the excitation leaks very slowly out of the medium.

The equation of motion for coherence $S_{1}^{\dagger}$ contains a loss term (due to isotropic spontaneous emission) and a linear gain term (due to emission into bright polariton). The two can compensate each other. However the linear phase-insensitive amplification is also accompanied by correspondingly increased fluctuations, represented by new Langevin forces $\tilde{F}_{D}(t), \tilde{F}_{S_{1}}^{\dagger}(t)$. Terms resulting from the direct coupling of dark and bright polaritons can be neglected for $\left|\Omega_{2}^{2}\right| \gg \gamma_{a g} \kappa$, which is reminiscent of the condition under which EIT is established.

To quantify the resulting quantum correlations we introduce a squeezing parameter in direct analogy to the optical parametric case. We define the quadratures $X_{1}=\left(S_{1}+S_{1}^{\dagger}\right) / \sqrt{2}, Y_{1}=i\left(S_{1}-S_{1}^{\dagger}\right) / \sqrt{2}$; these can be measured e.g. by converting spin excitations to light.
Correlations between the modes appear due to dynamical evolution and squeezing is found in the quadratures of the sum and difference modes $X_{ \pm}=\left(X_{1} \pm X_{2}\right) / \sqrt{2}$ and $Y_{ \pm}=\left(Y_{1} \pm Y_{2}\right) \sqrt{2}$. For small number of excitations the sum and difference modes obey standard commutation relations $\left[X_{\alpha}, Y_{\beta}\right]=-i \delta_{\alpha, \beta}$ where $\alpha, \beta=+,-$ or 1,2 . A quadrature $Y_{ \pm}$is squeezed when $\Delta Y_{ \pm}(t)^{2}<1 / 2$.

We find that squeezing is optimal under conditions of four-photon resonance $\left(\delta_{1}=\delta_{2}\right)$ and in the limit of $\eta \gg 1$ (Fig.3). Evolution leads to maximum squeezing of $Y_{+}$at $t=t^{*}$ after which the growing fluctuations in $X_{+}$give rise to increased noise in $Y_{+}$. Note that the number of excitations grows exponentially with time (Fig.3c). Specifically, in the case $g_{1}=g_{2}$, for $\xi t>1$, we have:

$$
\begin{aligned}
\Delta Y_{+}(t)^{2} & =1 / 2\left\{e^{-2 \xi t}+2 \frac{\gamma_{L}}{\xi}+\frac{\kappa / \eta}{\xi}\right. \\
& \left.+e^{2 \xi t}\left(\frac{\gamma_{L}+\kappa / \eta}{4 \xi}\right)^{2}\right\}
\end{aligned}
$$

where we have neglected terms of higher order in $\gamma_{L} / \xi$ and $\kappa / \xi$. The maximum amount of squeezing is obtained after an interaction time $t^{*}$ such that $e^{-2 \xi t^{*}}=$ $\left(\gamma_{L}+\kappa / \eta\right) / 4 \xi$ and is given by $\Delta Y_{+}^{2}=\left(5 \gamma_{L}+3 \kappa / \eta\right) / 4 \xi$. Since both the interaction parameter $\xi$ and the relaxation rate of the polariton $\gamma_{D}=\gamma_{L}+\kappa / \eta$ depend on the single photon detuning $\Delta$ (Fig.3a), we find that squeezing is optimized for the single-photon detuning $\Delta_{o p t}=\gamma_{a g} \sqrt{\frac{5\left|\Omega_{1}\right|^{2}}{3\left|\Omega_{2}\right|^{2}} \frac{\left|g_{2}\right|^{2} N}{\gamma_{a g} \kappa}}$, and

$$
\Delta Y_{\text {+opt }}^{2}=\frac{\sqrt{15 / 4}}{\sqrt{\left|g_{2}\right|^{2} N / \gamma_{a g} \kappa}} .
$$

Note that the denominator is equal to the atomic density-length product multiplied by an empty cavity finesse and can easily exceed $10^{4}$ even for modest values of the density-length product and cavity finesse. Note also that the strong coupling regime of cavity QED $g \geq \max [\kappa, \gamma]$ is not required to achieve strong correlations, in fact as long as $g^{2} N \geq 2 \kappa \gamma$ squeezing is achieved. Furthermore, although a cavity configuration was used for simplicity, the results of the present analysis remain qualitatively valid in the limit of unity finesse, i.e. free space. We further emphasize that typical generation rate resulting in such optimal squeezing $\Omega_{1} \Omega_{2} / \Delta_{\text {opt }}$ can easily be on the order of fraction of $\mathrm{MHz}$. In such a case other decoherence mechanisms are negligible. Doppler shifts can also be disregarded as long as all fields are co-propagating.

To summarize, we have presented a scheme based on the interaction of coherent classical light with an optically dense ensemble of atoms that leads to effective coherent spin-changing interactions involving pairs of atoms. We have shown that this process is robust with respect to realistic decoherence mechanisms and can result in rapid generation of correlated (spin squeezed) 
atomic ensembles. Furthermore, the resulting spin excitations can be easily converted into photons on demand, which facilitates applications in quantum information processing. Possible applications involving highprecision measurements in atomic clocks can be also foreseen. We further note that extension of this work into the domain of very large atomic density-length product or high-finesse cavities might allow to create maximally spin-squeezed states or macroscopic quantum superpositions ("Schrodinger cat" states). This in turn might allow to observe interaction-induced quantum phase transitions [22].

We thank M.Fleischhauer, J.I.Cirac, V.Vuletic, S.Yelin and P.Zoller for helpful discussions. This work was supported by the NSF through the grant to the ITAMP. L.M.D. acknowledges support from the Austrian and Chinese Science Foundation.

[1] M.A. Nielsen and I.L. Chuang., Quantum computation and quantum information, (Cambridge University Press, New York, 2000).

[2] D. Bouwmeester, A.K. Ekert, A. Zeilinger (eds.), The physics of quantum information, (Springer, New York, 2000).

[3] D.J. Wineland, et al., Phys. Rev. A 46, R6797 (1992); ibid 50, 67 (1994). S. F. Huelga, et al. Phys. Rev. Lett. 79, 3865 (1997).

[4] H. Touchette and S. Lloyd, Phys. Rev. Lett. 84, 1156 (2000)

[5] L. V. Hau et al., Nature 397, 594 (1999); M. Kash et al. Phys. Rev. Lett. 82, 5229 (1999); D.Budker et al., ibid 83, (1999).

[6] M.D. Lukin et al., Phys. Rev. Lett. 84, 4232 (2000); M. Fleischhauer and M.D. Lukin, Phys. Rev. Lett. 84, 5094 (2000).

[7] C. Liu et al., Nature 409, 490 (2001); D.F. Phillips et al., Phys. Rev. Lett. 86, 783 (2001).

[8] D.F. Walls and G.J. Milburn, Quantum Optics (Springer, Berlin, 1994).

[9] J.I. Cirac et al., Phys. Rev. Lett. 78, 3221 (1997); S.J. Enk et al., Science 279, 205 (1998).

[10] L.-M. Duan et al., quant-ph/0105105.

[11] P.Meystre, Atom Optics, (Springer, New York, 2001).

[12] J. Stenger et al., Nature 396, 345 (1998).

[13] D.J. Heinzen et al, Phys. Rev. Lett. 84, 5029 (2000).

[14] L.-M. Duan et al., Phys. Rev. Lett. 85, 3991 (2000); A. Sørensen et al., Nature 409,63 (2001).

[15] A. Kuzmich et al., Phys. Rev. Lett. 79, 4782 (1997); J. Hald, et al., ibid 1319 (1999).

[16] A. Kuzmich, L. Mandel and N.P. Bigelow, Phys. Rev. Lett. 85, 1594 (2000).

[17] I. Bouchoule and K. Mølmer, quant-ph/0105144

[18] J.D. Franson and T.B. Pittman, Phys. Rev. A 60, 917 (1999).
[19] T. Opatrný and G. Kurizki, Fortschr. Phys. 481125 (2000); J.D. Franson, Fortschr. Phys. 48, 1133 (2000); M. Fleischhauer, quant-ph/0007042.

[20] M. Fleischhauer and M.D. Lukin, quant-ph/0106066

[21] M. Kitagawa and M. Ueda, Phys. Rev. A 47, 5138 (1993).

[22] S.Sachdev, Quantum phase transitions, (Cambridge University Press, New York, 1999).
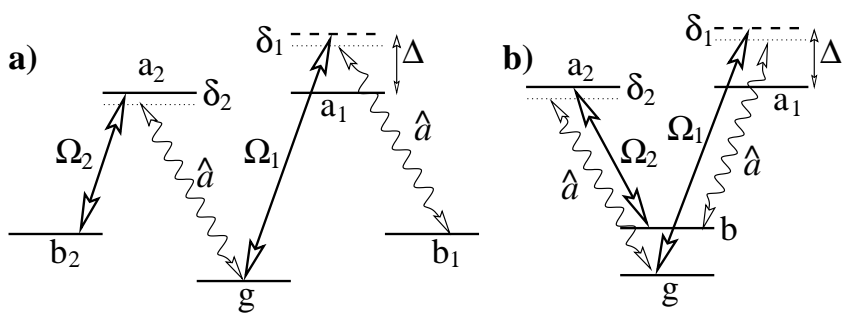

FIG. 1. Level scheme for the coherent interaction leading to pairs of atoms in (a) different final states $\left|b_{2}\right\rangle$ and $\left|b_{1}\right\rangle$, (b) the same final state $|b\rangle$.

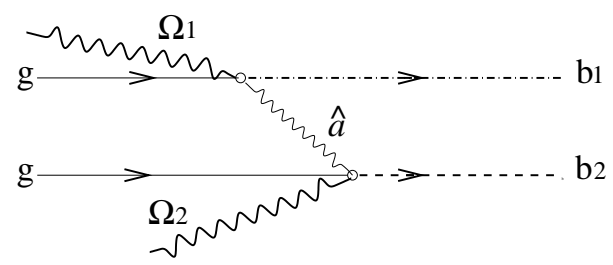

FIG. 2. Diagram illustrating coherent atom-atom interaction mediated by dark-state polariton, leading to the creation of a pair of spin-flipped atoms. a)

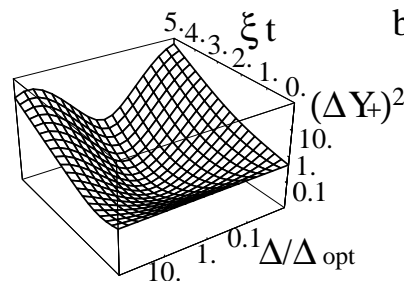

c)

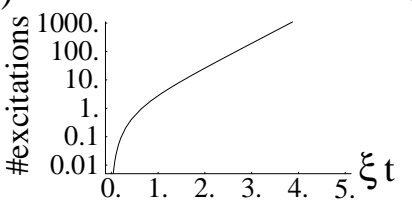

b) $\left(\Delta \mathrm{Y}_{+}\right)^{2}$

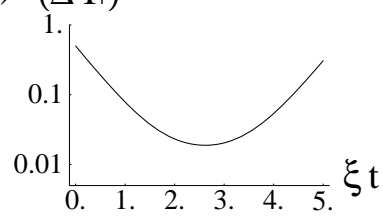

d) $(\Delta Y+)^{2}$

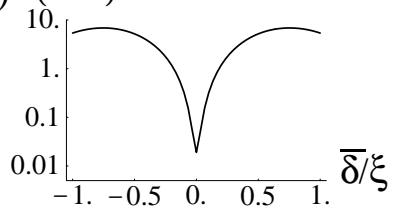

FIG. 3. (a) Quadrature variance $\Delta Y_{+}^{2}$ vs. single-photon detuning $\Delta$ and interaction time $\xi t$, (b) same for $\Delta=\Delta_{\text {opt }}$ and $\delta_{1}=\delta_{2}$ showing maximum squeezing $\Delta Y_{+}^{2} \simeq 0.02$ (for $\left.\sqrt{g_{2}^{2} N / \gamma_{a g} \kappa}=100\right)$, (c) Number of excitations pumped in the system vs. time (same conditions as in b) and (d) $\Delta Y_{+}\left(t^{*}\right)^{2}$ vs. two-photon detuning $\bar{\delta} \equiv\left(\delta_{1}-\delta_{2}\right) / 2$ for $\Delta=\Delta_{o p t}$ and where $t^{*}$ gives maximum squeezing. 